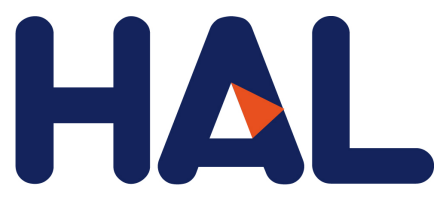

archives-ouvertes

\title{
Resolving Segmental Polymer Dynamics in Nanocomposites by Incoherent Neutron Spin-Echo Spectroscopy
}

Dafne Musino, Julian Oberdisse, Bela Farago, Angel Alegria, Anne-Caroline Genix

\section{To cite this version:}

Dafne Musino, Julian Oberdisse, Bela Farago, Angel Alegria, Anne-Caroline Genix. Resolving Segmental Polymer Dynamics in Nanocomposites by Incoherent Neutron Spin-Echo Spectroscopy. ACS Macro Letters, Washington, D.C: American Chemical Society, 2020, 9 (6), pp.910-916. 10.1021/acsmacrolett.0c00369 . hal-02908124

\section{HAL Id: hal-02908124 \\ https://hal.archives-ouvertes.fr/hal-02908124}

Submitted on 28 Jul 2020

HAL is a multi-disciplinary open access archive for the deposit and dissemination of scientific research documents, whether they are published or not. The documents may come from teaching and research institutions in France or abroad, or from public or private research centers.
L'archive ouverte pluridisciplinaire HAL, est destinée au dépôt et à la diffusion de documents scientifiques de niveau recherche, publiés ou non, émanant des établissements d'enseignement et de recherche français ou étrangers, des laboratoires publics ou privés. 


\title{
Resolving segmental polymer dynamics in nanocomposites by incoherent neutron spin-echo spectroscopy
}

\author{
Dafne Musino $^{1}$, Julian Oberdisse ${ }^{1}$, Bela Farago ${ }^{2}$, Angel Alegria ${ }^{3}$, Anne-Caroline Genix ${ }^{1 *}$ \\ ${ }^{1}$ Laboratoire Charles Coulomb (L2C), Université de Montpellier, CNRS, F-34095 Montpellier, \\ France \\ ${ }^{2}$ Institut Max von Laue-Paul Langevin (ILL), 71 Avenue des Martyrs, CS 20156, F-38042 Grenoble \\ Cedex 9, France \\ ${ }^{3}$ Departamento de Fisica de Materiales (UPV/EHU) and Materials Physics Center (CSIC- \\ UPV/EHU), Paseo Manuel Lardizábal 5, San Sebastian 20018, Spain \\ *Author for correspondence: anne-caroline.genix@umontpellier.fr
}

\begin{abstract}
The segmental dynamics of styrene-butadiene nanocomposites with embedded silica nanoparticles (NPs, ca. 20\%v) has been studied by broadband dielectric (BDS) and neutron spin-echo spectroscopy (NSE). It is shown by BDS that overlapping contributions only allow to conclude on a range of distributions of relaxation times in simplified industrial nanocomposites formed with highly polydisperse NPs. For comparison, structurally similar but less aggregated colloidal nanocomposites have a well-defined distribution of relaxation times due to the reduced influence of interfacial polarization processes. This distribution is widened with respect to the neat polymer, without change in the position of the maximum, and at most a small slowing down visible in the average time. We then demonstrate that incoherent NSE can be used to resolve small modifications of segmental dynamics of the industrial samples. By carefully choosing the q-vector of the measurement, experiments with fully hydrogenated polymer give access to the self-dynamics of the polymer in the presence of silica on the scale of approximately $1 \mathrm{~nm}$. Our high resolution measurements show that the segmental motion is slightly but systematically slowed down also by the presence of the industrial filler NPs.
\end{abstract}


Nanocomposites made by the dispersion of hard nanoscale fillers in soft polymer matrices are fundamentally intriguing materials because of their outstanding rheology. ${ }^{1-4}$ They serve in technological applications, such as car tires. ${ }^{5-6}$ The mechanical response of polymer nanocomposites (PNC) is determined by microscopic structure and dynamics. ${ }^{7-8}$ The former is governed by the concentration and dispersion of the filler, e.g., individually dispersed ${ }^{9-10}$ or strongly aggregated nanoparticles (NPs), ${ }^{11}$ possibly with large length-scale structure, ${ }^{12}$ while the latter depends on the properties and quantity of the available polymer-filler interface, i.e., it is indirectly related to structure. For instance, the average NP size sets the specific surface available for polymer-NP interactions. Moreover, the large-scale distribution of filler in the matrix may be heterogeneous, i.e., dense zones may offer locally more contact and confinement of the polymer than a well-dispersed and homogeneous distribution. To quantify these aspects, the structure of polymer nanocomposites is usually characterized by transmission electron microscopy (TEM), ${ }^{13-16}$ and in particular small-angle X-ray or neutron scattering (SAXS or SANS), ${ }^{17}$ combined with appropriate modelling approaches, ${ }^{18-21}$ possibly including simulations. ${ }^{22-23}$

The dynamical properties of PNCs may be studied by broadband dielectric spectroscopy (BDS) ${ }^{24-27}$ or NMR. ${ }^{28-29}$ These techniques average over the macroscopic response of the samples to external fields, without spatial resolution. Low-field solid-state NMR allows the identification of polymer zones possessing a slowed-down, immobilized, or possibly glassy dynamics. ${ }^{30-31}$ Recently, a simplified industrial systems defined below has been investigated by BDS. ${ }^{32-33}$ By following the evolution of interfacial polarization processes related to water and ionic conduction along the polymer-filler interface, the percolation of the filler across the sample with volume fraction or grafting has been evidenced. Regarding the segmental $\alpha$-relaxation associated with the glass transition, data analysis was compatible with the assumption of polymer dynamics unperturbed by the filler up to $\Phi_{\mathrm{NP}}=20 \% \mathrm{v}$ silica volume fraction. However, due to masking of the BDS-signal by the intense interfacial processes, the effect of the filler on the $\alpha$-process could not be resolved conclusively. NMR provided evidence for the existence of a weak fraction of slowed-down polymer, ${ }^{28,33}$ which could correspond to a nanometric layer surrounding the filler. However, NMR does not give any indication on relevant length scales, and the slowing down cannot be directly traced back to molecular mechanisms.

Quasi-elastic neutron scattering (QENS) and in particular neutron spin-echo (NSE) spectroscopy allows to directly probe polymer dynamics at a molecular level with space and time resolution. ${ }^{34-35}$ In protonated nanocomposites, QENS spectra measured by backscattering yield the incoherent scattering function and give the self-motions of $\mathrm{H}$ atoms. Slowing down of the segmental dynamics has been found for aggregated PNCs with adsorbed chains on the NP surface. ${ }^{36}$ The collective motions associated with segmental relaxation are probed in fully deuterated (D) samples by NSE (coherent scattering). ${ }^{37}$ NSE also allows to observe the single-chain dynamic structure factor and the reptation process, using H/D- 
contrast between chains. ${ }^{38-41}$ However, the use of deuterated chains is hardly feasible for industrial mixing processes necessitating large quantities. It is shown here for the first time that incoherent NSE obtained with purely hydrogenated samples allows accessing the local segmental dynamics on the scale of approximately a nanometer in polymer nanocomposites, without perturbation by the coherent silica scattering.

To advance our understanding of the origin of macroscopic nanocomposite properties, we attempt here to resolve the impact of silica on the segmental dynamics of virtually monodisperse linear styrenebutadiene (SB) chains in highly loaded simplified industrial nanocomposites by NSE $\left(\Phi_{\mathrm{NP}}=18.3 \% \mathrm{v}\right.$, $\mathrm{MW}=177 \mathrm{~kg} / \mathrm{mol}, \mathrm{PI}=1.02, \mathrm{~T}_{\mathrm{g}}=-25^{\circ} \mathrm{C}$, dried two days under vacuum at room temperature). The system is formulated ${ }^{18,42}$ by solid-phase mixing of the polymer with a powder of precipitated silica pellets of industrial origin (Zeosil@ 1165MP from Solvay, $\mathrm{R}_{0}=8.55 \mathrm{~nm}$, log-normal polydispersity $\sigma=$ $0.27, \mathrm{~V}_{\mathrm{NP}}=3.610^{3} \mathrm{~nm}^{3}$ ), ${ }^{18}$ without any curing additives but the typical coating agent (octeo), granting them the term 'simplified'. The structure and rheology of such simplified industrial nanocomposites have been investigated previously. ${ }^{18,43}$ For comparison, we have set up a model system using the same $\mathrm{SB}$ polymer, but with well-defined colloidal silica (Ludox TM40, $\mathrm{R}_{0}=12.5 \mathrm{~nm}, \sigma=0.12, \mathrm{~V}_{\mathrm{NP}}=8.710^{3}$ $\left.\mathrm{nm}^{3}\right)$. This colloidal PNC sample $\left(\Phi_{\mathrm{NP}}=20.4 \% \mathrm{v}\right)$ is prepared by casting from a common solvent (MEK) as detailed elsewhere, ${ }^{44}$ using bare NPs.

We have started our study with BDS measurements (Novocontrol Alpha) of the industrial nanocomposite, and the real and imaginary parts of the permittivity, $\varepsilon^{\prime}(\omega)$ and $\varepsilon^{\prime \prime}(\omega)$, are shown in Figure 1a at $\mathrm{T}=273 \mathrm{~K}$. One can clearly see, in the dielectric loss, a strong and broad peak associated with a Maxwell-Wagner-Sillars (MWS) process, which results in the trapping and accumulation of charges at the polymer/silica interface. It is therefore structure dependent. ${ }^{33,45} \mathrm{On}$ the high-frequency side of this peak, a weak hump remains visible for the $\alpha$-process. Additional data at temperatures ranging from 253 to $278 \mathrm{~K}$ are given in SI showing that all processes are shifted towards higher frequencies with increasing temperature. The dielectric data for neat SB are also given in SI. 

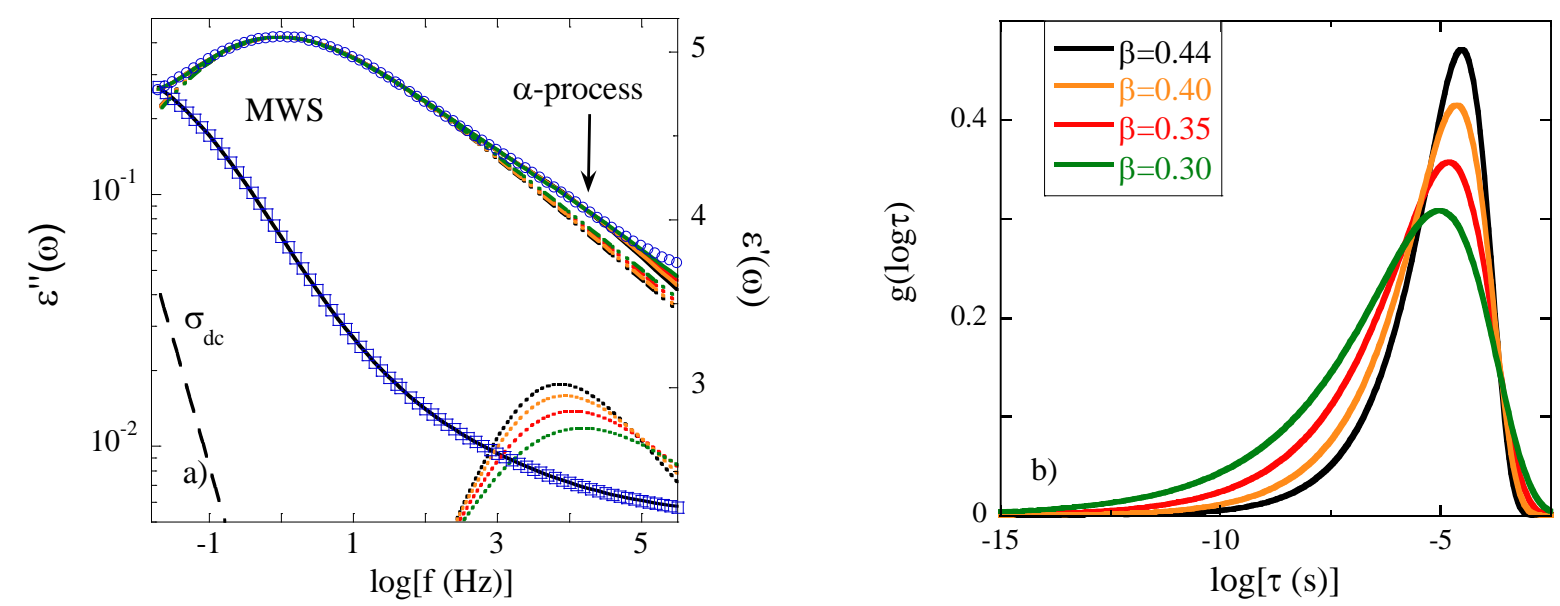

Figure 1: a) Frequency dependence of the imaginary (circles) and real (squares) parts of the complex dielectric permittivity measured on industrial PNC with ca. $20 \% \mathrm{v}$ silica at $\mathrm{T}=273 \mathrm{~K}$. Lines are fits to the experimental data by a purely dissipative dc-conductivity term (dashed line) and two HN-functions: interfacial MWS process (dashed-dotted lines) and $\alpha$-process (dotted lines). For the later, four different spectral shapes associated with the KWW distributions given in b) are shown to reproduce the $\alpha$-process. b) KWW distributions of relaxation times for different $\beta$ parameters. $\beta=0.44$ and 0.35 correspond to the parameters obtained for the pure SB matrix and the colloidal PNC, respectively.

Due to the strong overlap of the MWS-process with the $\alpha$-process, the shape and position of the latter could not be unambiguously determined. As shown previously, ${ }^{33}$ it is possible to describe the data in Figure 1a using the same Havriliak-Negami (HN) function as obtained with the neat SB matrix for the $\alpha$-process modulo a trivial correction for the dielectric strength (black dotted line). For consistency with the NSE data discussed below, a stretched exponential or Kohlrausch-William-Watts (KWW) function has been used here to describe the segmental dynamics in time domain. This implies that the HN-shape parameters in frequency domain are coupled (see SI for details), and the $\alpha$-time scale is the only free fit parameter in the $\mathrm{HN}$-function for a given stretching exponent, $\beta$. In Figure 1, we used a KWW function with $\beta=0.44$ as obtained from the analysis of neat SB (see SI), but equivalently good descriptions of the industrial PNC data in Figure 1a could be obtained using larger distributions of $\alpha$-relaxation times up to $\beta=0.30$ as illustrated in Figure $1 \mathrm{~b}$. There is thus a range of compatible distribution functions describing the segmental dynamics, without the possibility of discriminating between them. One can use the average characteristic time, $\langle\tau\rangle=\tau_{\mathrm{W}} \Gamma(1 / \beta) / \beta$, as a representative time scale for comparison of functions with different spectral shapes. ${ }^{46}$ For the industrial PNC sample, $\log \langle\tau\rangle$ lies in the range between -4.19 and -4.37 , whereas -4.22 was found for the silica-free matrix. If any, this suggest only a minor modification of the time scale upon addition of silica.

The origin of the weak signature of segmental relaxation in industrial nanocomposites lies in the superposition with strong MWS processes, which are related to aggregation and NP connectivity in this sample. We have therefore compared to an equivalent model PNC with the same silica content but well- 
defined colloidal NPs. This colloidal system has a similar structure, with less developed aggregation, as can be seen in Figure 2a using SAXS (beamline SWING, SOLEIL, Saint Aubin, France).
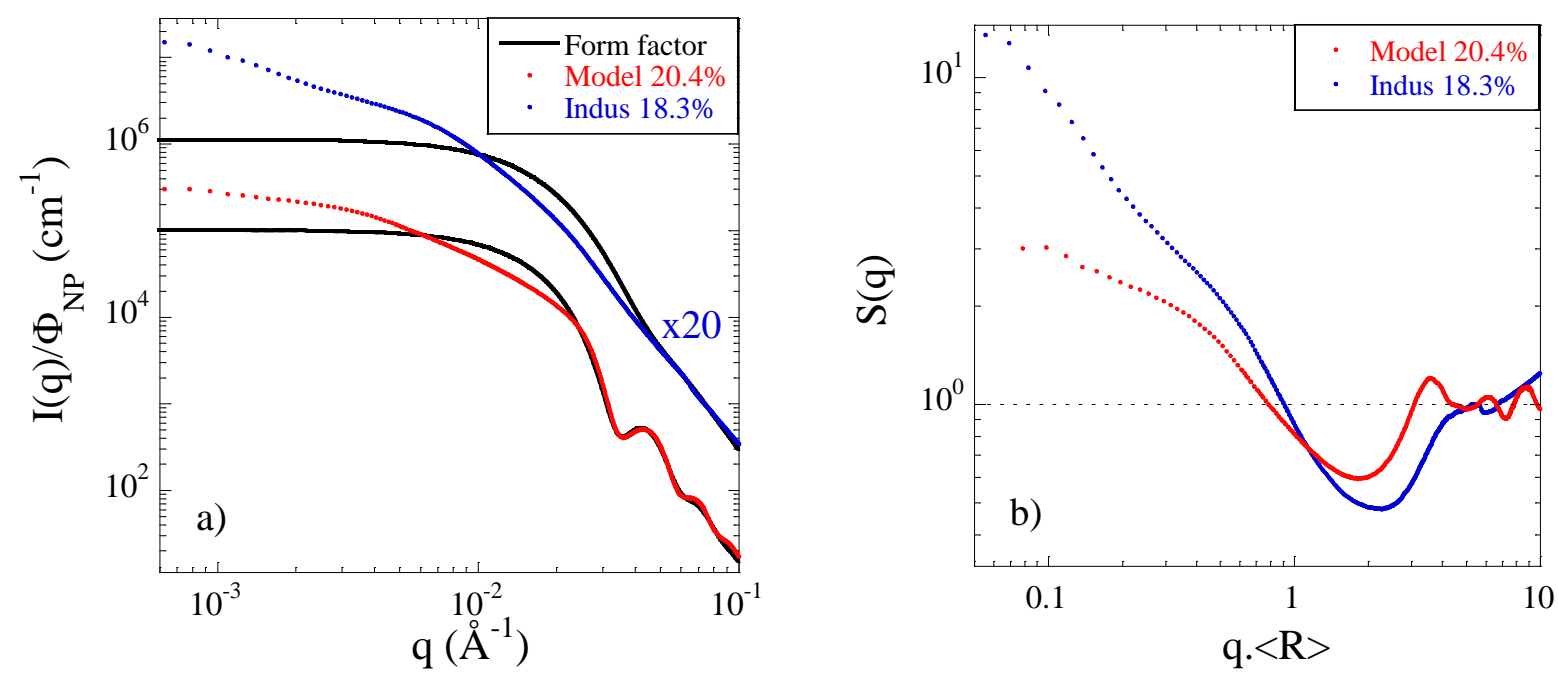

Figure 2: a) Structural comparison by SAXS. Industrial (blue) and model (red) PNCs with the corresponding NP form factor (black) measured in dilute conditions and reported in silica-polymer contrast. Silica volume fractions are given in the legend, and intensities are shifted for clarity. b) Apparent structure factor S(q) obtained by division of the intensities given in a) by their respective form factors.

The SAXS intensities in Figure 2a highlight the structural differences. At large q, the intensity decay follows a Porod law close to $\mathrm{q}^{-4}$, with a prefactor $2 \pi \Delta \rho^{2} \mathrm{~S} / \mathrm{V}$ related to identical silica-polymer contrast $\Delta \rho$, and to the specific surface $\mathrm{S} / \mathrm{V}$. The NPs have been chosen to have a comparable size and $\mathrm{S} / \mathrm{V}$, which is why curves have been shifted for clarity in Figure 2a. In the intermediate q-range, the model colloidal particles display damped oscillations indicating a lower polydispersity than the industrial ones, in agreement with the particle size distribution. At low q, finally, a stronger increase of the intensity of the industrial PNC is observed, indicating higher levels of aggregation.

By comparing the intensities to their respective NP form factor also shown in Figure 2a, both curves display a correlation hole - a dip with respect to the form factor - at $q>10^{-2} \AA^{-1}$, related to different local NP densities. ${ }^{47}$ To compensate for the different particle size, we have plotted in Figure $2 b$ the apparent structure factor S(q) obtained by dividing the PNC-intensities by their average form factor as a function of $\mathrm{q}\langle\mathrm{R}\rangle$. The deeper correlation hole around $\mathrm{q}\langle\mathrm{R}\rangle=2$ indicates locally denser NP assemblies for industrial PNCs. This is accompanied by the stronger low-q increase of $S(q)$ in the latter case. Such a feature points towards a higher aggregation, and, given the high average density of some $20 \% \mathrm{v}$, a higher structural heterogeneity on large scales, in agreement with our structural modelling of aggregates populating branches of higher density. ${ }^{18}$ As a result, the existence of denser zones - the fractal branches - leads to locally higher concentrations, more particle contacts, and thus more dominant interfacial polarization processes favored by NP connectivity. On the contrary, in absence of any mechanical solid- 
phase mixing of pre-aggregated powders, the model PNCs are more homogeneous. They also form percolating paths at high concentration, as described in detail recently ${ }^{22}$, which serve as framework for - albeit weaker - MWS processes.

The BDS data of the colloidal system are shown in Figure 3 at $\mathrm{T}=273 \mathrm{~K}$ (other temperatures are given in SI): interfacial MWS-processes with lower dielectric strength are shifted towards lower frequency, resulting in a weaker contribution in this frequency range, and we can clearly distinguish and trustfully describe the $\alpha$-process. Here, we found that it is not possible to use the matrix shape parameter $(\beta=$ 0.44 , see SI for details), and the best fit yields $\beta=0.35$. A distribution with this shape has been included in Figure 1b: it is located in the middle of the possible range of the industrial sample. As with the industrial sample, the position of the HN-maximum is unchanged with respect to the neat polymer (see SI), but due to the broadening of the time distribution the average $\log \langle\tau\rangle=-4.10$ indicates a slight slowing down.

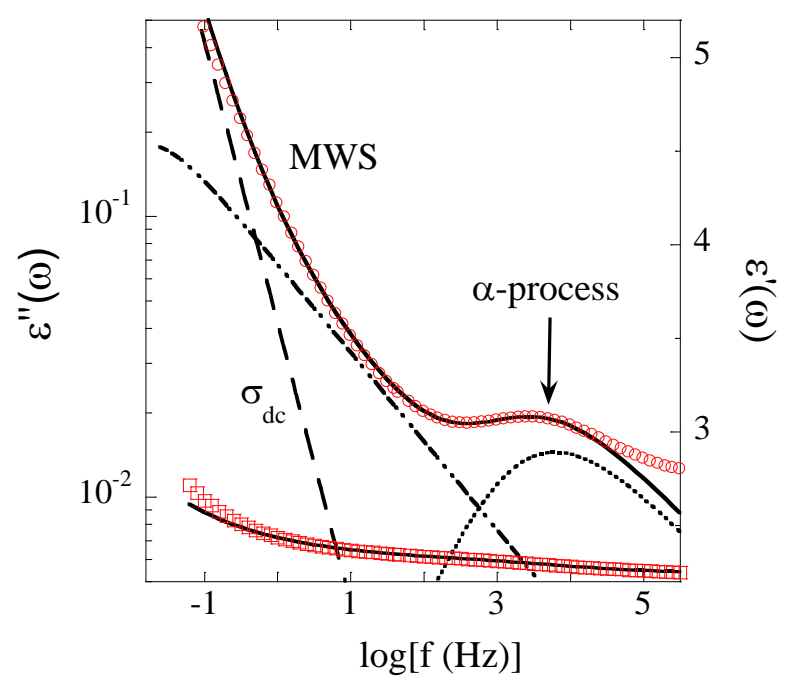

Figure 3: Frequency dependence of the imaginary (circles) and real (squares) parts of the complex dielectric permittivity measured on colloidal PNC with ca. $20 \% \mathrm{v}$ silica at $\mathrm{T}=273 \mathrm{~K}$. Lines are fits to the experimental data by a purely dissipative dc-conductivity term (dashed line) and two $\mathrm{HN}$-functions: interfacial MWS process (dashed-dotted line) and $\alpha$-process (dotted line).

The comparison between the two preceding analyses of industrial and colloidal samples shows that contrary to colloidal nanocomposites it is difficult to probe the impact of (industrial) filler interfaces on segmental motion by BDS. We thus investigate the dynamics of industrial nanocomposites by NSE.

Neutron spin echo spectroscopy offers quantitative insight into the dynamical processes of glassforming systems. We study the hydrogen self-correlation on protonated simplified industrial nanocomposites. This is challenging as NSE is ordinarily used to probe coherent scattering. For incoherent dynamics, the signal is reduced to $1 / 3$ due to the $2 / 3$ probability of flipping the scattered 
neutron spin by incoherent scattering events, see eq 1. To the best of our knowledge, incoherent NSE measurements have never been attempted before with PNCs.

$$
I_{\text {NSE }}(q, t)=\frac{\frac{1}{3} I_{\text {inc }}(q, t)-I_{\text {coh }}(q, t)}{\frac{1}{3} I_{\text {inc }}(q, 0)-I_{\text {coh }}(q, 0)}
$$

The first step is to define the proper q-range for NSE, i.e., values of q which are high enough to make the coherent contribution of the silica negligible. We have thus performed WAXS measurements shown in Figure 4. Due to a similar weighting of the atomic correlations, X-ray measurements are identical to static neutron scattering, besides a general prefactor. It is clear from Figure 4 that the coherent contribution of the silica is strongly reduced above $0.2 \AA^{-1}$, where the contribution of the polymer which scatters most of the neutrons incoherently dominates. Above $0.6 \AA^{-1}$, an increase towards a (coherent) scattering peak associated with correlations between molecular SB groups is observed, as in the pure polymer. This defines the optimal q-range for incoherent NSE: between 0.2 and $0.6 \AA^{-1}$, as indicated by the square in Figure 4. This q-range is further confirmed by SANS experiments on the same samples reported in the SI.

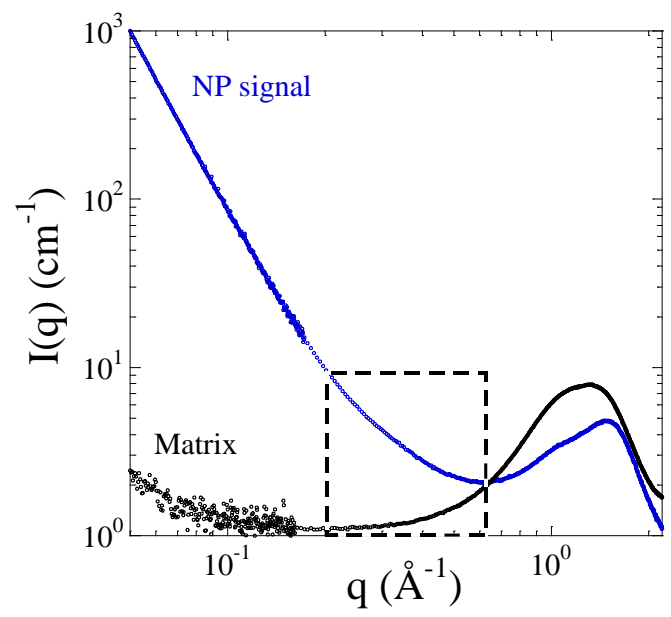

Figure 4: WAXS intensity of simplified industrial PNC (blue) and the neat SB polymer (black). The dashed box illustrates the optimal q-range for neutron spin echo experiments. Note that WAXS only shows the coherent scattering, while for neutrons a strong incoherent scattering is added; our optimum q-range is determined by minimizing the coherent contributions.

NSE was performed on the spectrometer IN15 (ILL, Grenoble, France, $\lambda=6 \AA$ ). Experimental resolution was corrected by normalizing echo amplitudes to a purely elastic scatterer. The comparison of the incoherent scattering functions at $100^{\circ} \mathrm{C}$ for the pure matrix and the industrial PNC at $\mathrm{q}=0.2 \AA^{-1}$ is shown in Figure 5a. The salient feature is that the matrix decays to zero, as expected well-above the calorimetric glass-transition where thermal motion induces loss of monomer self-correlation. For the nanocomposite, $\mathrm{I}_{\mathrm{NSE}}(\mathrm{q}, \mathrm{t})$ becomes negative at long times at this q-vector. From the polarization of the scattered beam, we know that the incoherent contribution dominates at short times, but the elastic contribution to $I_{\text {coh }}(q, t)$ may outweigh the decaying $I_{\text {inc }}(q, t)$ at long times in eq $1: I_{N S E}(q, t)$ can thus 
become negative. This is observed in Figure 5a, where the industrial PNC turns negative because the immobilized silica still contributes at this q-value. Above $\mathrm{q}=0.28 \AA^{-1}$, this contribution becomes weak. For industrial PNCs, we have therefore measured the self-correlation at different q-values up to $0.54 \AA^{-}$ ${ }^{1}$, as shown in Figure $5 b$.
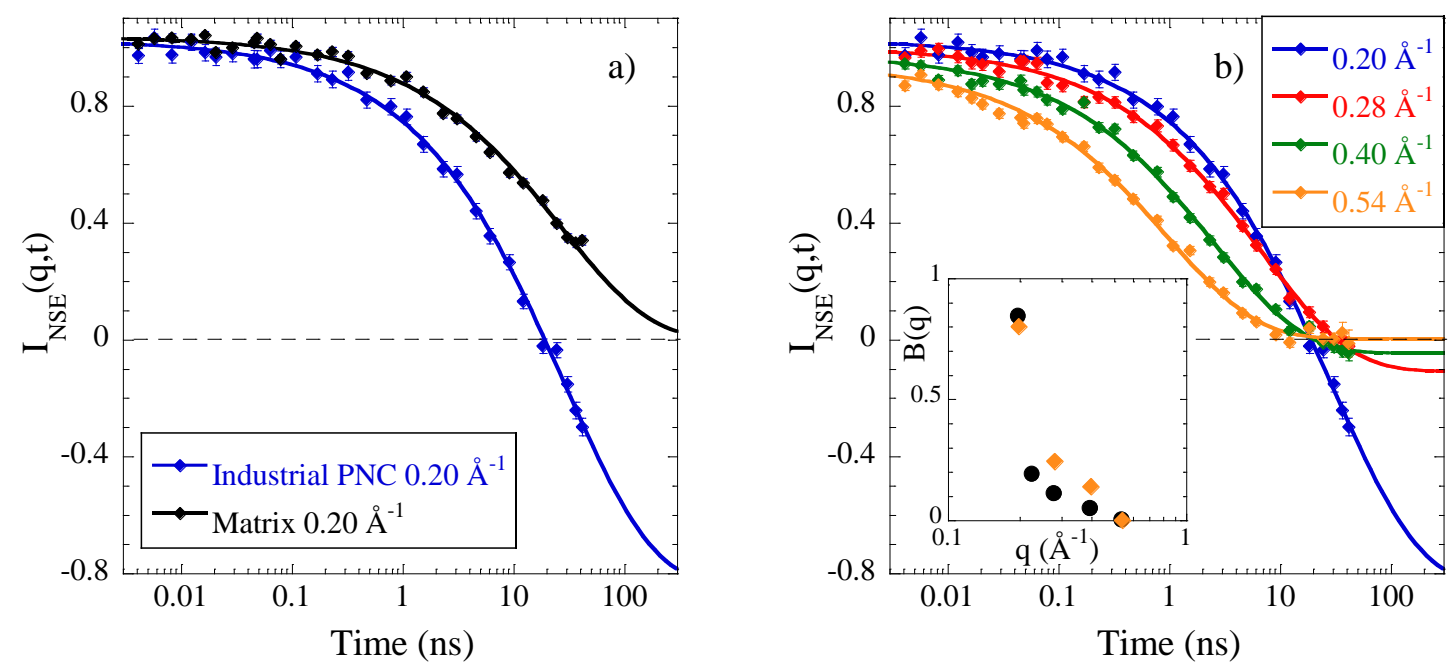

Figure 5: Incoherent scattering functions of SB protons at $\mathrm{T}=100^{\circ} \mathrm{C}$. a) Matrix (black) and industrial PNC with $20 \% \mathrm{v}$ of silica (blue) at $0.20 \AA^{-1}$. b) Industrial PNC at several q values. Lines are fits with eq (2). The inset shows the decrease of the elastic contribution $\mathrm{B}(\mathrm{q})$ as a function of $\mathrm{q}$ (circles), compared to an independent determination of the coherent silica part to the intensity (diamonds), demonstrating that the elastic $\mathrm{B}(\mathrm{q})$ is fully coherent.

The intermediate scattering functions have been described using the same KWW stretched exponential as for BDS, adding a q-dependent constant B(q) to account for the elastic plateau in NSE:

$$
\frac{\mathrm{I}(\mathrm{q}, \mathrm{t})}{\mathrm{I}(\mathrm{q}, 0)}=\mathrm{A}(\mathrm{q}) \exp \left[-\left(\frac{\mathrm{t}}{\tau_{\mathrm{w}}}\right)^{\beta}\right]-\mathrm{B}(\mathrm{q})
$$

All matrix and PNC-data could be fitted with a single $\beta$-parameter of 0.55 , close to 0.5 reported for polymers, ${ }^{34}$ indicating that dynamical heterogeneities are not modified in presence of silica. This result is in line with BDS (Figure 1a) where the $\alpha$-relaxation may be described by the unchanged matrix contribution. The elastic level $\mathrm{B}(\mathrm{q})$ is strongly reduced with increasing $\mathrm{q}$ as highlighted in the inset of Figure $5 \mathrm{~b} . \mathrm{B}(\mathrm{q})$ has a negative contribution from the (coherent) silica contributions and partial positive contribution due to immobilized (incoherent) polymer. It has been compared to an estimation of the coherent part in the inset. The reasonable agreement implies that the polymer contribution to the elastic level is close to zero. There is thus no measurable evidence of an immobilized polymer fraction within the NSE window, in agreement with NMR results where only a few percent were found for the immobilized polymer fraction in this sample. It is however possible that the dynamics is modified as we will see below. 
The evolution of the characteristic times $\tau_{\mathrm{w}}$ versus $\mathrm{q}$ has been determined from the self-correlation functions in Figure 5, and is reported in the SI (Figure S6). For both the pure matrix and the nanocomposite, we found that the q-dependence of $\tau_{\mathrm{w}}$ is compatible with a power law with an exponent $2 / \beta$ typical for Gaussian behavior. The time scales in the two samples appeared to be similar with and without silica, but the uncertainty induced by the a priori unknown elastic level $\mathrm{B}(\mathrm{q})$ impedes any conclusion on small shifts in segmental dynamics. Only at the highest q-vector, the correlation in the nanocomposite clearly decays to $\mathrm{B}(\mathrm{q})=0$ within the NSE window (Figure $5 \mathrm{~b}$ ). We have thus concentrated our efforts on the highest $q$ value $\left(0.54 \AA^{-1}\right)$, and varied the temperature. Self-correlation functions obtained for the industrial PNC at three temperatures are shown in Figure 6a together with its matrix.
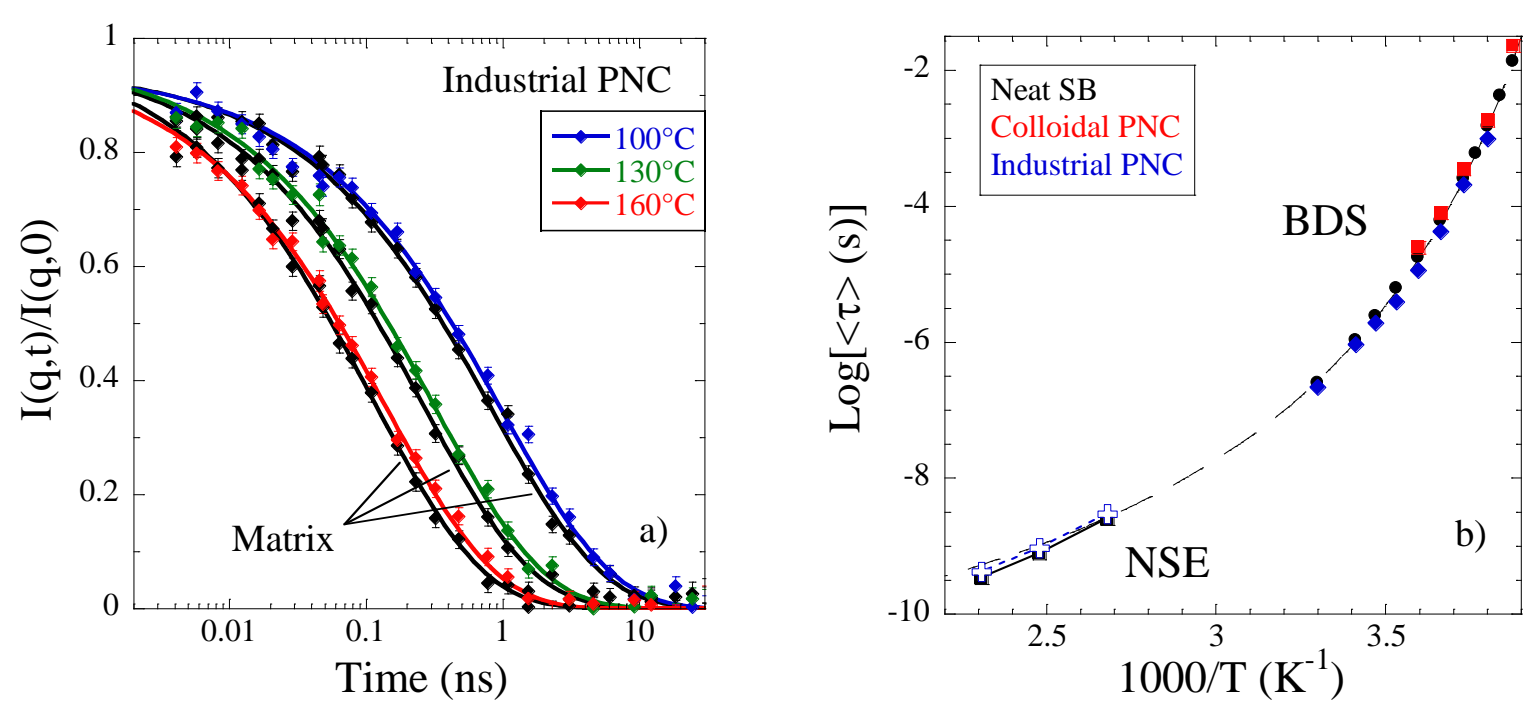

Figure 6: a) NSE data at $q=0.54 \AA^{-1}$ for the matrix (black) and the industrial PNC measured at different temperatures. Lines are fits using eq 2 with $\mathrm{B}(\mathrm{q})=0$ and $\beta=0.55$. b) Relaxation map with the characteristic average times for segmental relaxation from NSE data $\left(q=0.54 \AA^{-1}\right)$ and BDS for the two PNC samples and neat $\mathrm{SB}$. For the industrial PNC, the same shape parameter as for the matrix has been used in the analysis of the BDS spectra for consistency with NSE. The Vogel-Fulcher-Tammann (VFT) fit of the BDS times for the pure polymer matrix is represented by a dashed line.

The self-correlations in Figure 6a represent to our knowledge the first observation by incoherent NSE showing a systematic shift towards longer times in industrial nanocomposites, meaning that the segmental dynamics is slower in presence of silica. Although the shift is small, it is furthermore confirmed by an intermediate shift observed in a sample of intermediate concentration ( $8.3 \% \mathrm{v}$, see SI). One may also observe that the data in Figure 6a overlap at short and long times, while the strong decay at intermediate times is shifted, giving credit to the absence of any normalization-related vertical shift. Finally, describing the PNC-curves with the matrix curves is not compatible with the error bars (see SI). Our results are summarized on a relaxation map where the average time scales are plotted as a function of $1 / \mathrm{T}$ in Figure $6 \mathrm{~b}$. Over many orders of magnitude, the difference in time scale obtained in the 
industrial PNC appears to be small, demonstrating that the impact of silica even in strongly aggregated systems remains rather small at these high temperatures $\left(T \geq T_{g}+125 \mathrm{~K}\right)$. NSE at the probed nanometric length-scale evidences segmental motions that are in the same range than the VFT-extrapolated ${ }^{48}$ macroscopic BDS response of the matrix. For comparison, the time scales obtained by BDS with the colloidal PNC are also included in Figure 6b: as commented above, a slight slowing down of the dynamics is also observed in this case and it is systematic with $\mathrm{T}$, whereas uncertainty on the shape distribution impedes any clear conclusion for the industrial PNC by BDS, which appears to have slightly lower relaxation times here due to the use of the matrix fit parameters.

The main result of this article is that we have investigated the segmental relaxation of highly loaded polymer nanocomposites. In the lower temperature range, by BDS, we have shown that due to the influence of interfacial polarization, only a range of parameters and thus relaxation time distributions can be determined. There is broadening but essentially no shift in the peak position. The range in relaxation times encompasses the relaxation time distributions of the pure matrix, and of a colloidal PNC, indicating that if any, only minor modifications of the polymer dynamics are caused by the presence of the silica. Given the impossibility to conclude on the fate of the segmental relaxation in industrial PNCs, we have then demonstrated for the first time the feasibility of resolving the chain dynamics by incoherent NSE measurements. A weak but systematic shift on the nanometric scale $(\sim 2 \pi / \mathrm{q})$ towards longer times at high $\mathrm{T}$ is observed, and the decay can be described with the same KWW functional shape as the matrix. This slowing down is probably caused by the confinement of the polymer within dense zones and thus in contact with the NP interface. Also, the spatial resolution of NSE, here probing ca. $1 \mathrm{~nm}$, shows that the slowing down takes indeed place close to the filler interface. To conclude, it is hoped that our high-resolution measurements contribute to deeper understanding of the striking dynamical and in particular rheological properties of polymer nanocomposites, which result from a subtle interplay between polymer dynamics at the filler interface and filler microstructure.

\section{Associated Content.}

Supporting Information available: Complementary BDS results and data treatment, SANS data, and complementary NSE results

Acknowledgements. The authors are thankful for support by the ANR NANODYN project, grant ANR14-CE22-0001-01 of the French Agence Nationale de la Recherche. We thank Philippe DieudonnéGeorge (L2C) and Thomas Bizien (Soleil) for WAXS and SAXS measurements, respectively. The polymer was a gift from Synthos, and Radoslaw Kozak, Nathalia Meissner, and Pawel Weda (Synthos) are thanked for polymer synthesis. Discussions with Laurent Guy and Thomas Chaussée (Solvay) are gratefully acknowledged. 


\section{References}

(1) Kumar, S. K.; Benicewicz, B. C.; Vaia, R. A.; Winey, K. I., 50th Anniversary Perspective: Are Polymer Nanocomposites Practical for Applications? Macromolecules 2017, 50 (3), 714-731.

(2) Jancar, J.; Douglas, J. F.; Starr, F. W.; Kumar, S. K.; Cassagnau, P.; Lesser, A. J.; Sternstein, S. S.; Buehler, M. J., Current Issues in Research on Structure-Property Relationships in Polymer Nanocomposites. Polymer 2010, 51 (15), 3321-3343.

(3) Heinrich, G.; Kluppel, M.; Vilgis, T. A., Reinforcement of Elastomers. Current Opinion in Solid State \& Materials Science 2002, 6 (3), 195-203.

(4) Zhang, Q.; Archer, L. A., Poly(Ethylene Oxide)/Silica Nanocomposites: Structure and Rheology. Langmuir 2002, 18 (26), 10435-10442.

(5) Manoharan, P.; Naskar, K., Exploring a Highly Dispersible Silica-Elastomer Composite for Tire Applications. J. Appl. Polym. Sci. 2016, 133 (24), 43531.

(6) Mark, J. E.; Erman, B.; Eirich, F. R., Science and Technology of Rubber. Academic Press: San Diego, 1994.

(7) Genix, A.-C.; Oberdisse, J., Structure and Dynamics of Polymer Nanocomposites Studied by XRay and Neutron Scattering Techniques. Current Opinion in Colloid \& Interface Science 2015, 20 (4), 293-303.

(8) Kim, D.; Srivastava, S.; Narayanan, S.; Archer, L. A., Polymer Nanocomposites: Polymer and Particle Dynamics. Soft Matter 2012, 8 (42), 10813-10818.

(9) Meth, J. S.; Zane, S. G.; Chi, C.; Londono, J. D.; Wood, B. A.; Cotts, P.; Keating, M.; Guise, W.; Weigand, S., Development of Filler Structure in Colloidal Silica-Polymer Nanocomposites. Macromolecules 2011, 44 (20), 8301-8313.

(10) Inoubli, R.; Dagreou, S.; Lapp, A.; Billon, L.; Peyrelasse, J., Nanostructure and Mechanical Properties of Polybutylacrylate Filled with Grafted Silica Particles. Langmuir 2006, 22 (15), 6683-6689.

(11) Jouault, N.; Vallat, P.; Dalmas, F.; Said, S.; Jestin, J.; Boue, F., Well-Dispersed Fractal Aggregates as Filler in Polymer-Silica Nanocomposites: Long-Range Effects in Rheology. Macromolecules 2009, 42 (6), 2031-2040.

(12) Akcora, P.; Liu, H.; Kumar, S. K.; Moll, J.; Li, Y.; Benicewicz, B. C.; Schadler, L. S.; Acehan, D.; Panagiotopoulos, A. Z.; Pryamitsyn, V.; Ganesan, V.; Ilavsky, J.; Thiyagarajan, P.; Colby, R. H.; Douglas, J. F., Anisotropic Self-Assembly of Spherical Polymer-Grafted Nanoparticles. Nat Mater 2009, 8 (4), 354-359.

(13) Conzatti, L.; Costa, G.; Castellano, M.; Turturro, A.; Negroni, F. M.; Gerard, J. F., Morphology and Viscoelastic Behaviour of a Silica Filled Styrene/Butadiene Random Copolymer. Macromol. Mater. Eng. 2008, 293 (3), 178-187. 
(14) Chevigny, C.; Dalmas, F.; Di Cola, E.; Gigmes, D.; Bertin, D.; Boué, F.; Jestin, J., PolymerGrafted Nanoparticles Nanocomposites: Dispersion, Grafted Chain Conformation, and Rheological Behaviour. Macromolecules 2011, 44 (1), 122-133.

(15) Le Strat, D.; Dalmas, F.; Randriamahefa, S.; Jestin, J.; Wintgens, V., Mechanical Reinforcement in Model Elastomer Nanocomposites with Tuned Microstructure and Interactions. Polymer 2013, 54 (5), 1466-1479.

(16) Bokobza, L.; Chauvin, J.-P., Reinforcement of Natural Rubber: Use of in Situ Generated Silicas and Nanofibres of Sepiolite. Polymer 2005, 46 (12), 4144-4151.

(17) Lindner, P.; Zemb, T., Neutrons, X-Ray and Light Scattering. North Holland, Elsevier: Amsterdam, 2002.

(18) Baeza, G. P.; Genix, A. C.; Degrandcourt, C.; Petitjean, L.; Gummel, J.; Couty, M.; Oberdisse, J., Multiscale Filler Structure in Simplified Industrial Nanocomposite Silica/SBR Systems Studied by SAXS and TEM. Macromolecules 2013, 46 (1), 317-329.

(19) Botti, A.; Pyckhout-Hintzen, W.; Richter, D.; Urban, V.; Straube, E.; Kohlbrecher, J., Silica Filled Elastomers: Polymer Chain and Filler Characterization in the Undeformed State by a SANSSAXS Approach. Polymer 2003, 44 (24), 7505-7512.

(20) Rishi, K.; Beaucage, G.; Kuppa, V.; Mulderig, A.; Narayanan, V.; McGlasson, A.; Rackaitis, M.; Ilavsky, J., Impact of an Emergent Hierarchical Filler Network on Nanocomposite Dynamics. Macromolecules 2018, 51 (20), 7893-7904.

(21) Schneider, G. J., Correlation of Mass Fractal Dimension and Asymmetry. J. Chem. Phys. 2009, 130 (23), 234912.

(22) Musino, D.; Genix, A.-C.; Chauveau, E.; Bizien, T.; Oberdisse, J., Structural Identification of Percolation of Nanoparticles. Nanoscale 2020, 12, 3907-3915.

(23) Genix, A.-C.; Bocharova, V.; Carroll, B.; Lehmann, M.; Saito, T.; Krueger, S.; He, L.; Dieudonné-George, P.; Sokolov, A. P.; Oberdisse, J., Understanding the Static Interfacial Polymer Layer by Exploring the Dispersion States of Nanocomposites. ACS Applied Materials \& Interfaces 2019, 11 (19), 17863-17872.

(24) Otegui, J.; Schwartz, G. A.; Cerveny, S.; Colmenero, J.; Loichen, J.; Westermann, S., Influence of Water and Filler Content on the Dielectric Response of Silica-Filled Rubber Compounds. Macromolecules 2013, 46 (6), 2407-2416.

(25) Holt, A. P.; Griffin, P. J.; Bocharova, V.; Agapov, A. L.; Imel, A. E.; Dadmun, M. D.; Sangoro, J. R.; Sokolov, A. P., Dynamics at the Polymer/Nanoparticle Interface in Poly(2Vinylpyridine)/Silica Nanocomposites. Macromolecules 2014, 47 (5), 1837-1843.

(26) Carroll, B.; Cheng, S.; Sokolov, A. P., Analyzing the Interfacial Layer Properties in Polymer Nanocomposites by Broadband Dielectric Spectroscopy. Macromolecules 2017, 50 (16), 61496163. 
(27) Fragiadakis, D.; Bokobza, L.; Pissis, P., Dynamics near the Filler Surface in Natural RubberSilica Nanocomposites. Polymer 2011, 52 (14), 3175-3182.

(28) Mujtaba, A.; Keller, M.; Ilisch, S.; Radusch, H. J.; Beiner, M.; Thurn-Albrecht, T.; Saalwächter, K., Detection of Surface-Immobilized Components and Their Role in Viscoelastic Reinforcement of Rubber-Silica Nanocomposites. ACS Macro Letters 2014, 3 (5), 481-485.

(29) Saalwächter, K., Microstructure and Molecular Dynamics of Elastomers as Studied by Advanced Low-Resolution Nuclear Magnetic Resonance Methods. Rubber Chem. Technol. 2012, 85 (3), 350-386.

(30) Papon, A.; Saalwächter, K.; Schäler, K.; Guy, L.; Lequeux, F.; Montes, H., Low-Field NMR Investigations of Nanocomposites: Polymer Dynamics and Network Effects. Macromolecules 2011, 44 (4), 913-922.

(31) Papon, A.; Montes, H.; Lequeux, F.; Oberdisse, J.; Saalwachter, K.; Guy, L., Solid Particles in an Elastomer Matrix: Impact of Colloid Dispersion and Polymer Mobility Modification on the Mechanical Properties. Soft Matter 2012, 8 (15), 4090-4096.

(32) Baeza, G. P.; Oberdisse, J.; Alegria, A.; Couty, M.; Genix, A. C., A High-Temperature Dielectric Process as a Probe of Large-Scale Silica Filler Structure in Simplified Industrial Nanocomposites. Phys. Chem. Chem. Phys. 2015, 17, 1660-1666.

(33) Baeza, G. P.; Oberdisse, J.; Alegria, A.; Saalwaechter, K.; Couty, M.; Genix, A.-C., Depercolation of Aggregates Upon Polymer Grafting in Simplified Industrial Nanocomposites Studied with Dielectric Spectroscopy. Polymer 2015, 73, 131-138.

(34) Richter, D.; Monkenbusch, M.; Arbe, A.; Colmenero, J., Neutron Spin Echo in Polymer Systems. Springer Berlin Heidelberg, 2005; Vol. 174, p 1-221.

(35) Colmenero, J.; Arbe, A., Recent Progress on Polymer Dynamics by Neutron Scattering: From Simple Polymers to Complex Materials. J. Polym. Sci. B Polym. Phys. 2013, 51 (2), 87-113.

(36) Roh, J. H.; Tyagi, M.; Hogan, T. E.; Roland, C. M., Space-Dependent Dynamics in 1,4Polybutadiene Nanocomposite. Macromolecules 2013, 46 (16), 6667-6669.

(37) Ashkar, R.; Abdul Baki, M.; Tyagi, M.; Faraone, A.; Butler, P.; Krishnamoorti, R., Kinetic Polymer Arrest in Percolated SWNT Networks. ACS Macro Letters 2014, 3 (12), 1262-1265.

(38) Senses, E.; Ansar, S. M.; Kitchens, C. L.; Mao, Y.; Narayanan, S.; Natarajan, B.; Faraone, A., Small Particle Driven Chain Disentanglements in Polymer Nanocomposites. Phys. Rev. Lett. 2017, 118 (14), 147801.

(39) Nusser, K.; Schneider, G. J.; Richter, D., Microscopic Origin of the Terminal Relaxation Time in Polymer Nanocomposites: An Experimental Precedent. Soft Matter 2011, 7 (18), 7988-7991.

(40) Schneider, G. J.; Nusser, K.; Neueder, S.; Brodeck, M.; Willner, L.; Farago, B.; Holderer, O.; Briels, W. J.; Richter, D., Anomalous Chain Diffusion in Unentangled Model Polymer Nanocomposites. Soft Matter 2013, 9 (16), 4336-4348. 
(41) Senses, E.; Tyagi, M.; Natarajan, B.; Narayanan, S.; Faraone, A., Chain Dynamics and Nanoparticle Motion in Attractive Polymer Nanocomposites Subjected to Large Deformations. Soft Matter 2017, 13 (43), 7922-7929.

(42) Musino, D.; Genix, A.-C.; Fayolle, C.; Papon, A.; Guy, L.; Meissner, N.; Kozak, R.; Weda, P.; Bizien, T.; Chaussée, T.; Oberdisse, J., Synergistic Effect of Small Molecules on Large-Scale Structure of Simplified Industrial Nanocomposites. Macromolecules 2017, 50 (13), 5138-5145.

(43) Baeza, G. P.; Genix, A. C.; Degrandcourt, C.; Gummel, J.; Mujtaba, A.; Saalwächter, K.; ThurnAlbrecht, T.; Couty, M.; Oberdisse, J., Studying Twin-Samples Provides Evidence for a Unique Structure-Determining Parameter in Simplified Industrial Nanocomposites. ACS Macro Letters 2014, 3, 448-452.

(44) Musino, D.; Genix, A. C.; Chaussée, T.; Guy, L.; Meissner, N.; Kozak, R.; Bizien, T.; Oberdisse, J., Aggregate Formation of Surface-Modified Nanoparticles in Solvents and Polymer Nanocomposites Langmuir 2018, 34 (9), 3010-3020.

(45) Bohning, M.; Goering, H.; Fritz, A.; Brzezinka, K. W.; Turky, G.; Schonhals, A.; Schartel, B., Dielectric Study of Molecular Mobility in Poly(Propylene-Graft-Maleic Anhydride)/Clay Nanocomposites. Macromolecules 2005, 38 (7), 2764-2774.

(46) Arbe, A.; Colmenero, J., Characterization of the "Simple-Liquid" State in a Polymeric System: Coherent and Incoherent Scattering Functions. Phys. Rev. E 2009, 80 (4), 041805.

(47) Genix, A.-C.; Oberdisse, J., Determination of the Local Density of Polydisperse Nanoparticle Assemblies. Soft Matter 2017, 13 (44), 8144-8155.

(48) Kremer, F.; Schönhals, A., Broadband Dielectric Spectroscopy. Springer-Verlag: Heidelberg, 2003. 


\section{FOR TABLE OF CONTENTS USE ONLY}

Resolving segmental polymer dynamics in nanocomposites by incoherent neutron spin-echo spectroscopy

Dafne Musino, Julian Oberdisse, Bela Farago, Angel Alegria, Anne-Caroline Genix

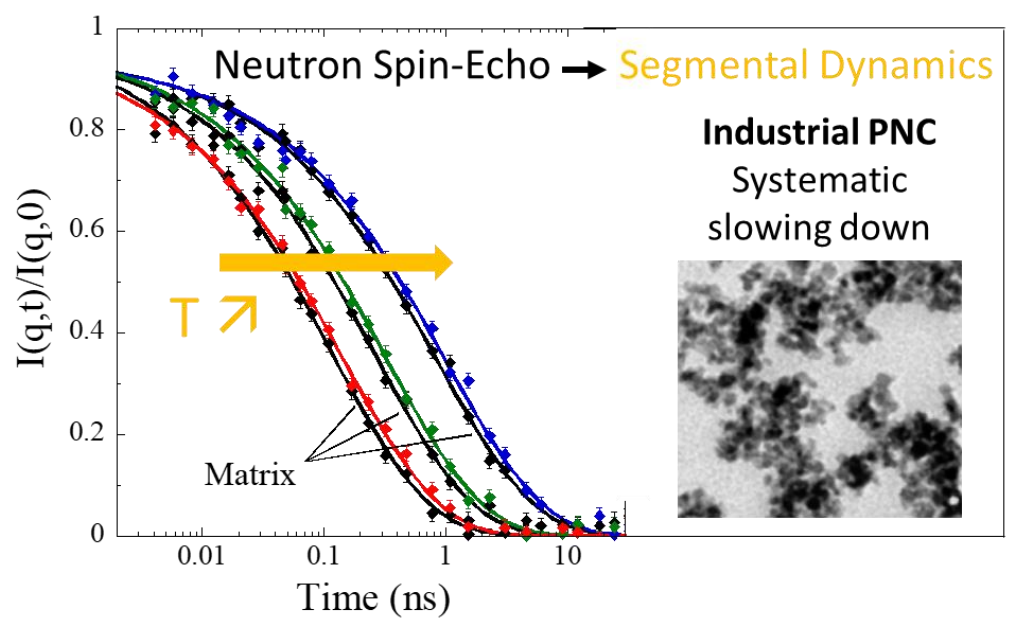

